\title{
Automated pupillometry and the FOUR score - what is the diagnostic benefit in neurointensive care?
}

\author{
Markus Harboe Olsen ${ }^{1}$ (D) Helene Ravnholt Jensen ${ }^{1}$ • Søren Røddik Ebdrup ${ }^{1}$ • Nina Hvid Topp ${ }^{1}$ • Ditte Gry Strange ${ }^{1}$. \\ Kirsten Møller ${ }^{1,2} \cdot$ Daniel Kondziella ${ }^{2,3}$
}

Received: 3 December 2019 / Accepted: 29 April 2020 / Published online: 7 May 2020

(C) Springer-Verlag GmbH Austria, part of Springer Nature 2020

\begin{abstract}
Introduction The Glasgow Coma Scale (GCS) and visual inspection of pupillary function are routine measures to monitor patients with impaired consciousness and predict their outcome in the neurointensive care unit (neuro-ICU). Our aim was to compare more recent measures, i.e. FOUR score and automated pupillometry, to standard monitoring with the GCS and visual inspection of pupils.

Methods Supervised trained nursing staff examined a consecutive sample of patients admitted to the neuro-ICU of a tertiary referral centre using GCS and FOUR score and assessing pupillary function first by visual inspection and then by automated pupillometry. Clinical outcome was evaluated 6 months after admission using the Glasgow Outcome ScaleExtended.

Results Fifty-six consecutive patients (median age 63 years) were assessed a total of 234 times. Of the 36 patients with at least one GCS score of 3, 13 had a favourable outcome. All seven patients with at least one FOUR score of $\leq 3$ had an unfavourable outcome, which was best predicted by a low "brainstem" sub-score. Compared to automated pupillometry, visual assessment underestimated pupillary diameters (median difference, $0.4 \mathrm{~mm} ; P=0.006$ ). Automated pupillometry detected a preserved pupillary light reflex in 10 patients, in whom visual inspection had missed pupillary constriction.

Discussion Training of nursing staff to implement frequent monitoring of patients in the neuro-ICU with FOUR score and automated pupillometry is feasible. Both measures provide additional clinical information compared to the GCS and visual assessment of pupillary function, most importantly a more granular classification of patients with low levels of consciousness by the FOUR score.
\end{abstract}

Keywords Consciousness · FOUR · GCS · Automated pupillometry $\cdot$ Prognostic marker

\section{Introduction}

Patients in the neurointensive care unit (neuro-ICU) often have impaired consciousness, either due to their brain injury

This article is part of the Topical Collection on Neurosurgical intensive care

Markus Harboe Olsen

oel@oelfam.com

1 Department of Neuroanaesthesiology, The Neuroscience Center, Rigshospitalet, University of Copenhagen, Copenhagen, Denmark

2 Faculty of Health and Medical Sciences, University of Copenhagen, Copenhagen, Denmark

3 Department of Neurology, Rigshospitalet, Copenhagen University Hospital, Copenhagen, Denmark or because of iatrogenic interventions such as sedation. Standardised evaluation is essential to monitor clinical improvement or deterioration and response to treatment. The widely used Glasgow Coma Scale (GCS) was originally introduced as a tool to assess levels of consciousness in patients with acute head trauma, but is now used in most patients with impaired consciousness [22]. However, the GCS has several limitations. Most importantly, its reliance on verbal output confounds the assessment of consciousness in patients with aphasia, the locked-in syndrome and those who are intubated $[1,24]$.

In contrast, the Full Outline of UnResponsiveness (FOUR) score does not rely on verbal assessment and includes a detailed assessment of brainstem function (Table 1) [24]. The FOUR score, introduced in 2005 [24], has been extensively tested for validity, reliability, reproducibility and prognostic 
value, primarily in patients with traumatic brain injury but also in a broader neuro-ICU population [2]. In general, in patients with impaired consciousness, the FOUR score allows a finer gradation of consciousness levels but has, primarily due to the missing verbal sub-score, a disadvantage in patients with only a minimally impaired consciousness.

In the same vein, visual inspection of pupillary function is a routine measure to monitor patients with impaired consciousness and predict their outcome [18], but its main disadvantage is the low interrater reliability [15], whereas automated infrared pupillometry using a handheld device allows for objective and quantitative measurement of pupillary function $[14,16]$.

In this feasibility study, we aimed to assess how the FOUR score and automated pupillometry add meaningful clinical information in a regular neuro-ICU setting, compared to GCS and visual inspection of pupils. To this end, we enrolled a consecutive cohort of patients admitted to the neuro-ICU and trained nursing staff to monitor patients using the FOUR score and automated pupillometry. These assessments were used to analyse (1) if the FOUR score results in a more granular evaluation of different levels of consciousness, (2) how well visual inspection of pupillary function reflects results from automated pupillometry and (3) if nursing staff can be trained to collect FOUR scores and perform automated pupillometry in a true-to-life neuro-ICU setting.

\section{Methods}

\section{Study population}

From June to September 2018, trained nursing staff examined a consecutive sample of patients with acute brain injury admitted to the neuro-ICU of a tertiary referral centre. Fiftysix consecutive patients ( 32 males, median age 63 years; IQR, 47-71; range, 19-86) with aneurysmal subarachnoid haemorrhage (32\%), intracerebral haematoma (27\%), traumatic brain injury $(25 \%)$ or other neurological or neurosurgical conditions (16\%) were assessed.

The nursing staff was taught the theoretical underpinnings and practical application of the FOUR score during 1 hour lectures, and they learned to assess pupillary diameters and light responses first by visual inspection and then by automated pupillometry (NPi200® NeurOptics pupillometer, Irvine, USA). Following this, the nursing staff assessed patients in the neuro-ICU under supervision before initiation of the main observational study period. Lectures and training were carried out by board-certified anaesthesiologists and a neurologist with expertise in neurocritical care. The Ethics Committee of the Capital Region waived the need for informed consent because data were collected as part of clinical routine (ref. number 18030137).

Patients were first dichotomized according to their lowest GCS score, so that one group consisted of patients with a GCS score of 3 at least once during ICU admission, and the other group comprised all other patients. We then dichotomized patients according to their pupillary function; i.e. patients who at least once had one or two non-responsive pupils were allocated to one group and the remaining patients to the other. Each pupil was counted as one assessment (i.e. two pupillary assessments per one assessment of consciousness level). Patients assessed more than 3 times were examined by at least two of the five trained nurses.

Clinical outcome was evaluated 6 months after admission, based on notes from electronic patient charts, using the Glasgow Outcome Scale-Extended (GOSE), and patients were again dichotomized according to their outcome (favourable, GOSE 5-8; unfavourable, GOSE 1-4). In
Table 1 Full Outline of UnResponsiveness (FOUR) score

Eye response
4 - eyelids open or opened, tracking, or blinking to
command
3 -eyelids open but not tracking
2 - eyelids closed but open to loud voice
1 - eyelids closed but open to pain
0 - eyelids remain closed with pain
Brainstem reflexes
4 - pupil and corneal reflexes present
3 - one pupil wide and fixed
2 - pupil or corneal reflexes absent
1 - pupil and corneal reflexes absent
0 - absent pupil, corneal, and cough reflex

Each component is summed up to a full score (i.e. from 0 to 16). The FOUR score was introduced by Widjicks et al. in 2005 [24]

\author{
Motor response \\ 4 - thumbs-up, fist, or peace sign \\ 3 - localizing to pain \\ 2 - flexion response to pain \\ 1 - extension response to pain \\ 0 - no response to pain or generalized myoclonus \\ status \\ Respiration \\ 4 - not intubated, regular breathing pattern \\ 3 - not intubated, Cheyne-Stokes breathing pattern \\ 2 - not intubated, irregular breathing \\ 1 - breathes above ventilator rate \\ 0 - breathes at ventilator rate or apnea
}


addition, we performed logistic regression to analyse GCS and FOUR scores without dichotomization.

\section{Literature search}

To interpret results from our observational study in the context of the medical literature, we did two systematic searches of PubMed and MEDLINE (March 25, 2020) with publications from 2005 and forward. The first search was a combination of "(FOUR or (Full Outline of UnResponsiveness))" and "pupillometry", which resulted in 65 entries. Following screening of abstracts, we did not identify any relevant publications. The second search combined "(FOUR or (Full Outline of UnResponsiveness $))$ " with "((Glasgow Coma Scale) OR (GCS))", resulting in 854 entries. Entries were screened for adult patients with acute brain injury in an intensive care setting, which yielded 26 relevant publications.

\section{Data handling and statistics}

Data handling and statistical analyses were done using $\mathrm{R}(\mathrm{R}$ 3.6.1, R Development Core Team [2008], Vienna, Austria). Univariate logistic regression was used to analyse the cut-off of FOUR score before a significant increase in GCS was observed. The absolute difference between visual evaluation and automated pupillometry for pupillary size was compared using the Student's $t$ test. Data are presented as either median, IQR and range or percentage, unless otherwise stated.

\section{Results}

Fifty-six patients were assessed a median of five times (IQR, 4-5; range, 1-9; total number of assessments, GCS/FOUR score, 234; visual inspection/automated pupillometry, 468). The minimum interval between assessments was $8 \mathrm{~h}$. Nineteen of the patients (34\%) achieved a favourable outcome $(\mathrm{GOSE} \geq 5$ ).

The median GCS was 6 (IQR, 3-8; range, 3-14), and the median FOUR score was 7 (IQR, 4-11; range, 0-16). Assessments with a GCS of 3 corresponded to a median FOUR score of 4 (IQR, 4-5; range, 0-6); similarly, GCS 35 corresponded to a median FOUR score of 4 (IQR, 4-5; range, $0-10$ ) (Fig. 1). GCS scores $>3$ were only obtained with FOUR scores of 6 or higher (Fig. 2). Of the 43 patients who at least once had a GCS score of 3-6, $13(35 \%)$ had a favourable outcome. In comparison, $31 \%$ of patients with GCS 7-14 achieved a favourable outcome as well (Table 2). In contrast, $39 \%$ of patients with a FOUR score $>3$ achieved a favourable outcome, whereas none of the 7 patients with at least one FOUR score of $\leq 3$ had a favourable outcome. Preserved brainstem reflexes seemed to be the main driver of favourable outcomes according to the FOUR score.
Visual inspection underestimated pupillary diameter as compared to automated pupillometry by $0.4 \mathrm{~mm}$ (IQR, 0.2 0.7 ; range, $0-3.4 ; p=0.006$, Wilcoxon signed-rank test) (Fig. 3a and b), whereas assessment of pupillary constriction by visual inspection was binary (present or absent), automated pupillometry provided quantitative data with an overall median pupillary constriction of $0.35 \mathrm{~mm}$ (IQR $0.15-0.68$; range $0-2.76)$. In 57 of the 86 pupils (66\%) where visual inspection suggested absence of pupillary constriction, automated pupillometry confirmed this observation (median constriction $0 \mathrm{~mm}$; IQR: 0-0.07; range: 0-0.38) (Fig. 3c). However, in the remaining 29 pupils (from 10 patients), where visual inspection suggested absence of pupillary constriction, automated pupillometry revealed a preserved pupillary light reflex. Furthermore, 7 pupils (from 5 patients), where visual inspection suggested intact pupillary constriction, did not constrict when examined with automated pupillometry. Visual inspection suggested absence of pupillary constriction at least once in 17 patients (88 pupils in total), 16 (94\%) of which had an unfavourable clinical outcome. Absence of pupillary constriction by automated pupillometry was noticed at least once in 15 patients (77 pupils), and 14 of them had an unfavourable outcome (93\%) (Table 2).

\section{Discussion}

Frequent bedside monitoring is essential to identify clinical deterioration in critically ill patients and to evaluate treatment responses. Our results suggest that the FOUR score and automated pupillometry provide additional clinical information and prognostic information compared to the GCS and visual inspection of the pupils, especially in assessments where the GCS score is 3. Moreover, we found that training of nursing staff is feasible to implement automated pupillometry and the FOUR score for frequent bedside monitoring in the ICU.

\section{Impaired consciousness}

A GCS score of 3 is considered less predictive of outcome [2]. Patients with a GCS of 3 for a prolonged period during their stay in the neuro-ICU are difficult to monitor for subtle changes in their clinical condition [2]. This may occur because a GCS of 3 is frequently associated with iatrogenic causes such as sedation rather than the underlying neurological condition. Indeed, in our study a GCS score of 3 was the most common result (40\% of assessments) and corresponded to a wide range of FOUR scores (from 0 to 6 ). These patients varied widely in terms of presence or absence of brainstem reflexes, which indicates that the FOUR score allows for a more granular classification of patients with severely impaired consciousness levels. 

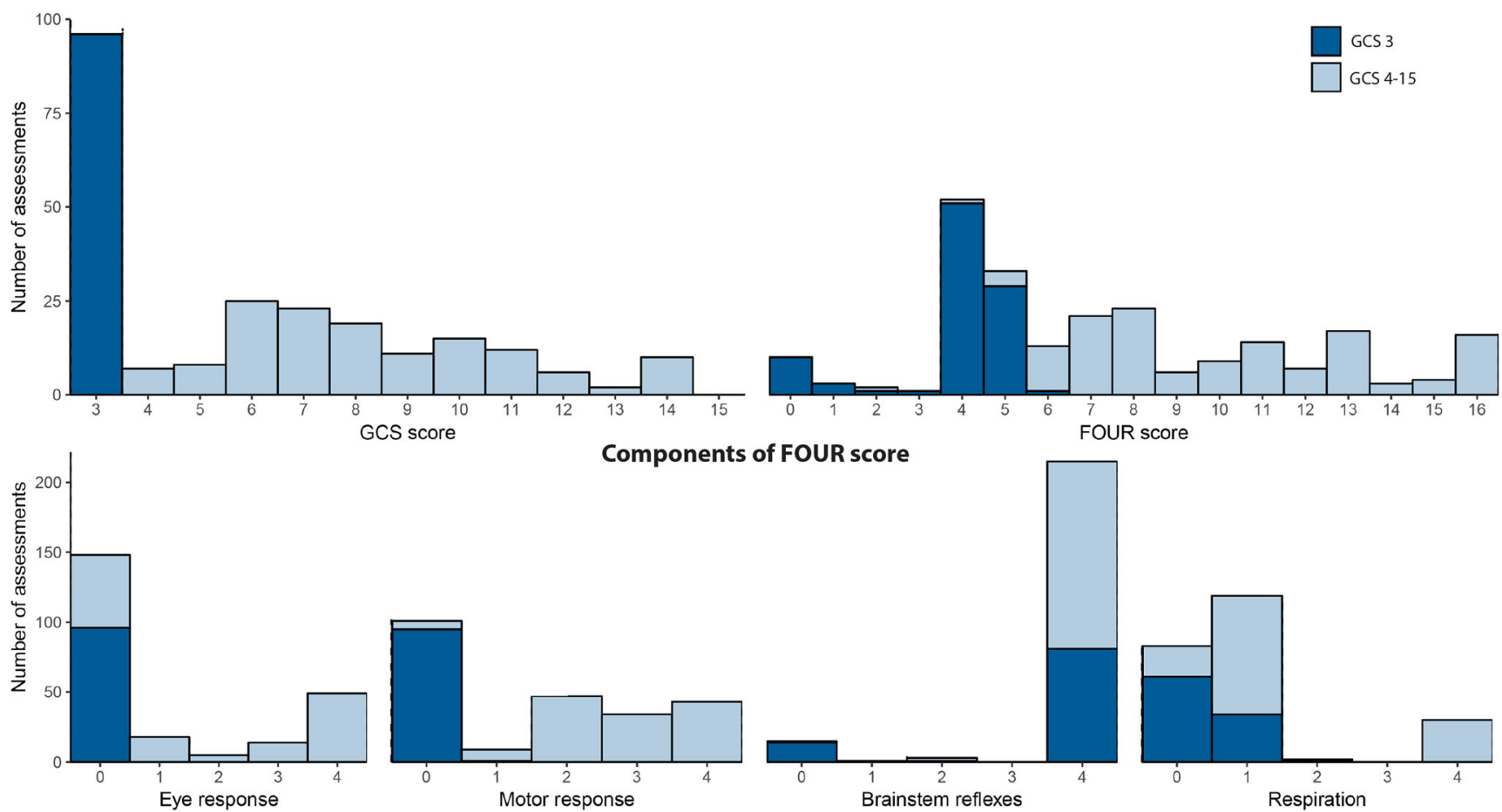

Fig. 1 Clinical assessments $(n=234)$ of 56 patients admitted to the neuro-ICU. Assessments of patients with a GCS score of 3 are depicted in dark blue. Upper row: Summarised GCS and FOUR scores. This graph shows that patients with a GCS score of 3 have a wide range of corresponding FOUR scores (from 0 to 6). Lower row: Subcomponents

of FOUR score. This graph shows that the finer distinction of patients with low consciousness levels by the FOUR score is mainly due to the sub-score "brainstem reflexes". GCS, Glasgow coma scale; FOUR, Full Outline of UnResponsiveness; GOSE, Glasgow outcome scale-extended

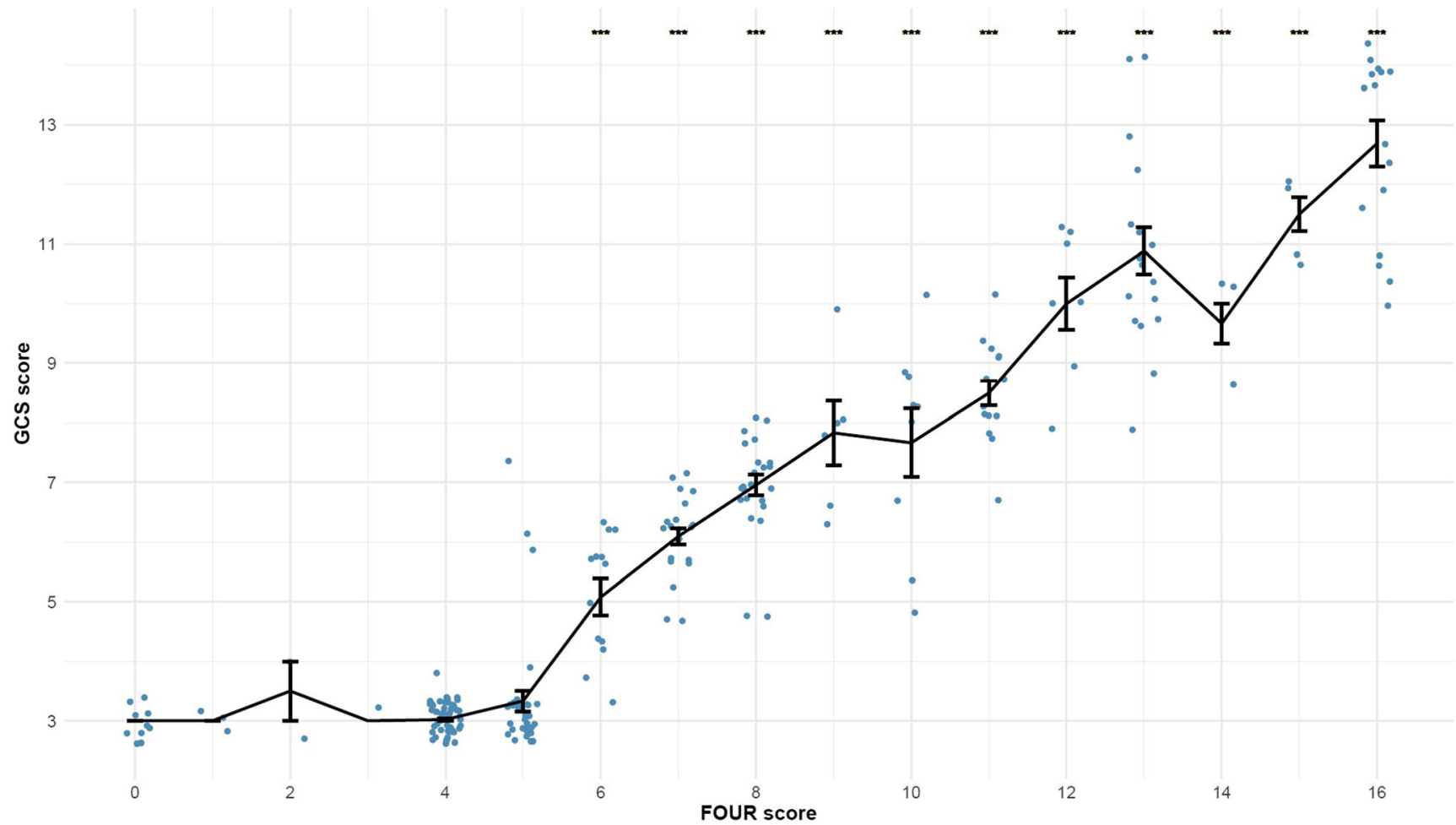

Fig. 2 Scatter plot illustrating the relationship between GCS score and FOUR score, again showing that patients with a GCS score of 3 may score very differently on the FOUR (from 0 to 6 ). The graph depicts mean and $95 \%$ confidence intervals of GCS scores for every FOUR score

(black line). Asterisks $(* * *)$ indicate significant difference $(p<0.0001)$ from baseline (FOUR score of 0 ) using logistic regression. GCS, Glasgow coma scale; FOUR, Full Outline of UnResponsiveness 
Table 2 Outcome categorized by lowest GCS and FOUR score

\begin{tabular}{llll}
\hline Measurement & Score & $N$ & Favourable outcome (GOSE $\geq 5)$ \\
\hline GCS & 3 & $36(64 \%)$ & $13(36 \%)$ \\
& $4-14$ & $20(36 \%)$ & $6(30 \%)$ \\
FOUR score & $0-3$ & $7(13 \%)$ & $0(0 \%)$ \\
& $4-16$ & $49(88 \%)$ & $19(39 \%)$ \\
FOUR score, eye response & $0-1$ & $49(88 \%)$ & $16(33 \%)$ \\
FOUR score, motor response & $2-4$ & $7(13 \%)$ & $3(43 \%)$ \\
& $0-1$ & $38(68 \%)$ & $14(37 \%)$ \\
FOUR score, brainstem reflexes & $2-4$ & $18(32 \%)$ & $5(28 \%)$ \\
& $0-1$ & $8(14 \%)$ & $1(12 \%)$ \\
FOUR score, respiration & $2-4$ & $40(71 \%)$ & $18(38 \%)$ \\
& $0-1$ & $51(91 \%)$ & $17(33 \%)$ \\
& $2-4$ & $5(9 \%)$ & $2(40 \%)$
\end{tabular}

This table shows that a very low FOUR score carries a much worse prognosis than a GCS of 3 . Restated, FOUR scores allow for a much finer distinction of patients with low levels of consciousness compared to the GCS. Data are presented as $n(\%) . N$, number of patients; GCS, Glasgow Come Score; FOUR, Full Outline of UnResponsiveness; GOSE, Glasgow Outcome Scale, Extended
The FOUR score has a high interrater reliability, which has been confirmed for patients with TBI $[3,12,13,20]$, as well as patients in general ICUs $[6,19,21]$ and neuro-ICUs $[8,10$, $26]$. The validity of the FOUR score renders it a good predictor of patient outcome. Validity studies have assessed the FOUR score at a specific time point during ICU admission to define the best cut-off between favourable and unfavourable outcome, showing that the FOUR score compares favourably as a predictor $[1,2,4,5]$.

Results from our cohort are well in line these findings, as no patient with a FOUR score of $\leq 3$ had a favourable outcome, while having a GCS of 3 was comparable with the rest of the cohort in terms of favourable outcome (36\% vs. $30 \%)$.
In contrast to most previous reports, we went further and frequently repeated assessments which allow us to conclude that more information can be obtained with repeated FOUR scores in patients with the lowest level of consciousness, as compared to the GCS. In other words, very low FOUR scores are more informative and carry a much more sinister prognosis than very low GCS scores.

\section{Pupillary function}

Frequent evaluation of pupillary function is valuable to monitor intracranial pressure (ICP) clinically. Evidence of fluctuations of pressure levels in certain intracranial compartments

\section{a Pupillary size}

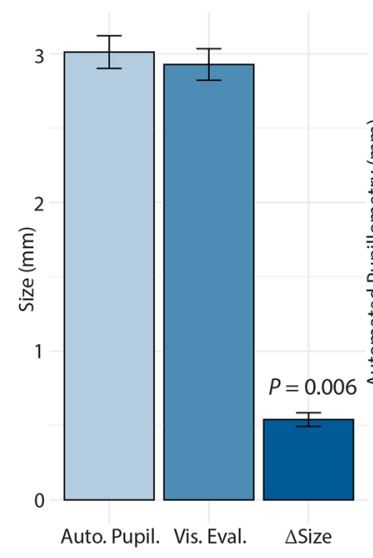

b Pupillary size comparison

8
Fig. 3 a Pupillary size and difference in size for every pupil assessed, i.e. the summarised numerical differences $(n=468)$. On average, visual inspection slightly underestimates pupillary size compared to automated pupillometry. b Comparison of every assessment of pupils assessed as $5 \mathrm{~mm}$ and below. This graph shows that in an individual patient, visual inspection may over- or underestimate pupillary sizes compared to

\section{Pupillary constriction}

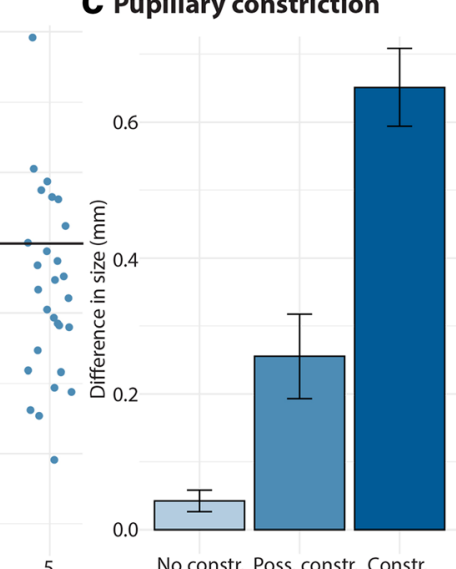

automated pupillometry. c Pupillary constriction recorded by automated pupillometry in $\mathrm{mm}$ categorized by conclusions from visual inspection. Although visual inspection can suggest absence of pupillary constriction, automated pupillometry may still show that pupils do constrict. Auto. Pupil., automated pupillometry; Vis. Eval., visual evaluation; $\Delta$ Size = difference in size measured; constr., constriction; Poss., possible 
(as opposed to global ICP), often missed by a monitoring pressure transducer, is sometimes revealed by pupillary function. Reliable estimations of pupillary size and contractibility are therefore needed. In this study, we showed that estimation of pupillary size and constriction by visual inspection can be very different from what is measured using automated pupillometry.

Like the FOUR score, pupillary constriction served as a valuable outcome predictor, irrespective of the mode of assessment. Automated pupillometry is more precise and can reveal pupillary constriction below $0.1 \mathrm{~mm}$ (in contrast to visual inspection), although the additional prognostic value in this study appeared relatively limited [11]. Still, whenever visual evaluation suggests that pupils appear not to constrict to light, automated pupillometry should be used to corroborate this, which would help avoiding wrong conclusions, including those related to prognosis. At the same time, it is important to be aware that automated pupillometry can occasionally miss a pupillary light reflex in very sluggish pupils [9]. Compared to visual inspection, however, automated pupillometry does provide detailed quantitative information such as the speed of pupillary constriction, the percentage change of pupillary diameters and dilation velocity $[7,14,16,23]$. The potential of these data as surrogate markers for the assessment of sedation, evolving brainstem damage, intracranial hypertension or consciousness levels should be investigated in future studies. Moreover, automated pupillometry might be valuable when it is mandatory to confirm the absence of pupillary function such as during brain death determination prior to organ donation.

To sum-up, our pupillary function data corroborate previous publications suggesting the overall superiority of automated pupillometry over visual evaluation of pupillary size in the neuro-ICU [17], although for clinical routine we would suggest using both methods as they complement each other. Restated, automated pupillometry should be added to visual inspection of pupils rather than replacing it.

\section{Limitations}

The major limitation of the present study is the low sample size. We pragmatically included a true-to-life, heterogenous cohort of patients with acute brain injury and did not adjust for factors such as sedation, disease aetiology or localization of brain damage. Since visual inspection of pupillary size and automated pupillometry were not obtained at the exact same timepoints (but for obvious reasons a few seconds apart), some of the difference between the methods could be artificial, e.g. because of changing ambient light intensity, although we made all efforts to exclude such confounders. Despite these caveats, we were able to show that the GCS has relatively limited value as a prognostic marker in the neuro-ICU and for monitoring subtle changes in the neurological status, as compared to the FOUR score, and that automated pupillometry adds valuable quantitative information as compared to visual inspection of pupils.

\section{Conclusion}

Training of nursing staff to implement frequent monitoring with the FOUR score and automated pupillometry is feasible in the neuro-ICU. Both measures provide additional clinical information compared to the GCS and visual inspection of pupils, in particular a much more granular distinction by the FOUR among unresponsive patients with a GCS score of 3 . This corroborates earlier data showing that the FOUR score performs better than the GCS for the prognostication of ICU mortality, probably because the respiratory and brainstem reflex components of the FOUR score reflect morbidity better than does the verbal part of the GCS [25] and, as stated previously, because low GCS scores are frequently iatrogenic, e.g. due to sedation and neuromuscular blocking agents. Finally, compared to visual inspection of pupils, automated pupillometry allows a much finer quantification of pupillary function, the clinical value of which needs to be addressed more in detail in future studies.

Authors' contributions $\mathrm{MHO}$ - concept and design of the study, analysis of data, important intellectual content, writing of the manuscript, approval of final manuscript; HRJ, SRE, NHT - data acquisition, important intellectual content, approval of final manuscript; DGS, KM-important intellectual content, approval of final manuscript; DK - concept and design of the study, analysis of data, important intellectual content, writing of the manuscript, approval of final manuscript.

\section{Compliance with ethical standards}

Conflict of interest All authors certify that they have no affiliations with or involvement in any organization or entity with any financial interest (such as honoraria; educational grants; participation in speakers' bureaus; membership, employment, consultancies, stock ownership, or other equity interest; and expert testimony or patent-licencing arrangements), or non-financial interest (such as personal or professional relationships, affiliations, knowledge or beliefs) in the subject matter or materials discussed in this manuscript.

Ethical approval All procedures performed in studies involving human participants were in accordance with the ethical standards of the institutional and national research committee and with the 1964 Helsinki declaration and its later amendments or comparable ethical standards.

\section{References}

1. Almojuela A, Hasen M, Zeiler FA (2019) The full outline of UnResponsiveness (FOUR) score and its use in outcome prediction: a scoping systematic review of the adult literature. Neurocrit Care 31(1):162-175 
2. Anestis DM, Tsitsopoulos PP, Tsonidis CA, Foroglou N (2020) The current significance of the FOUR score: a systematic review and critical analysis of the literature. J Neurol Sci 409:116600

3. Baratloo A, Shokravi M, Safari S, Aziz AK (2016) Predictive value of Glasgow Coma Score and Full Outline of Unresponsiveness score on the outcome of multiple trauma patients. Arch Iran Med 19(3):215-220

4. Chen B, Grothe C, Schaller K (2013) Validation of a new neurological score (FOUR Score) in the assessment of neurosurgical patients with severely impaired consciousness. Acta Neurochir 155(11):2133-2139 discussion 2139

5. Foo CC, Loan JJM, Brennan PM (2019) The relationship of the FOUR score to patient outcome: a systematic review. J Neurotrauma. https://doi.org/10.1089/neu.2018.6243

6. Iyer VN, Mandrekar JN, Danielson RD, Zubkov AY, Elmer JL, Wijdicks EFM (2009) Validity of the FOUR score coma scale in the medical intensive care unit. Mayo Clin Proc 84(8):694-701

7. Jahns F-P, Miroz JP, Messerer M, Daniel RT, Taccone FS, Eckert P, Oddo M (2019) Quantitative pupillometry for the monitoring of intracranial hypertension in patients with severe traumatic brain injury. Crit Care 23(1):155

8. Johnson VD, Whitcomb J (2013) Neuro/Trauma intensive care unit nurses' perception of the use of the full outline of unresponsiveness score versus the Glasgow Coma Scale when assessing the neurological status of intensive care unit patients. Dimens Crit Care Nurs 32(4):180-183

9. Kramer CL, Rabinstein AA, Wijdicks EFM, Hocker SE (2014) Neurologist versus machine: is the pupillometer better than the naked eye in detecting pupillary reactivity. Neurocrit Care 21(2): 309-311

10. McNett MM, Amato S, Philippbar SA (2016) A comparative study of Glasgow Coma Scale and Full Outline of Unresponsiveness scores for predicting long-term outcome after brain injury. J Neurosci Nurs 48(4):207-214

11. Meeker M, Du R, Bacchetti P, Privitera CM, Larson MD, Holland MC, Manley G (2005) Pupil examination: validity and clinical utility of an automated pupillometer. J Neurosci Nurs 37(1):34-40

12. Momenyan S, Mousavi SM, Dadkhahtehrani T, Sarvi F, Heidarifar R, Kabiri F, Mohebi E, Koohbor M (2017) Predictive validity and inter-rater reliability of the Persian version of Full Outline of Unresponsiveness among unconscious patients with traumatic brain injury in an intensive care unit. Neurocrit Care 27(2):229-236

13. Nyam T-TE, Ao K-H, Hung S-Y, Shen M-L, Yu T-C, Kuo J-R (2017) FOUR score predicts early outcome in patients after traumatic brain injury. Neurocrit Care 26(2):225-231

14. Obling L, Hassager C, Illum C, Grand J, Wiberg S, Lindholm MG, Winther-Jensen M, Kondziella D, Kjaergaard J (2019) Prognostic value of automated pupillometry: an unselected cohort from a cardiac intensive care unit. Eur Heart J Acute Cardiovasc Care: 2048872619842004

15. Olson DM, Stutzman S, Saju C, Wilson M, Zhao W, Aiyagari V (2016) Interrater reliability of pupillary assessments. Neurocrit Care 24(2):251-257

16. Peinkhofer C, Martens P, Grand J, Truelsen T, Knudsen GM, Kjaergaard J, Kondziella D (2018) Influence of strategic cortical infarctions on pupillary function. Front Neurol 9:916

17. Phillips SS, Mueller CM, Nogueira RG, Khalifa YM (2019) A systematic review assessing the current state of automated pupillometry in the NeuroICU. Neurocrit Care 31(1):142-161

18. Riker RR, Fugate JE, Participants in the International Multidisciplinary Consensus Conference on Multimodality Monitoring (2014) Clinical monitoring scales in acute brain injury: assessment of coma, pain, agitation, and delirium. Neurocrit Care 21(Suppl 2): S27-S37

19. Schnakers C, Majerus S, Giacino J et al (2008) A French validation study of the coma recovery scale-revised (CRS-R). Brain Inj 22(10):786-792

20. Sepahvand E, Jalali R, Mirzaei M, Ebrahimzadeh F, Ahmadi M, Amraii E (2016) Glasgow Coma Scale versus full outline of UnResponsiveness scale for prediction of outcomes in patients with traumatic brain injury in the intensive care unit. Turk Neurosurg 26(5):720-724

21. Singh TD, O’Horo JC, Gajic O, Sakusic A, Day CN, Mandrekar J, Kashyap R, Reddy DRS, Rabinstein AA (2018) Risk factors and outcomes of critically ill patients with acute brain failure: a novel end point. J Crit Care 43:42-47

22. Teasdale G, Jennett B (1974) Assessment of coma and impaired consciousness. A practical scale. Lancet 2(7872):81-84

23. Vassilieva A, Olsen MH, Peinkhofer C, Knudsen GM, Kondziella D (2019) Automated pupillometry to detect command following in neurological patients: a proof-of-concept study. PeerJ 7:e6929

24. Wijdicks EFMM, Bamlet WR, Maramattom BV, Manno EM, McClelland RL (2005) Validation of a new coma scale: the FOUR score. Ann Neurol 58(4):585-593

25. Wijdicks EFM, Kramer AA, Rohs T, Hanna S, Sadaka F, O’Brien J, Bible S, Dickess SM, Foss M (2015) Comparison of the Full Outline of UnResponsiveness score and the Glasgow Coma Scale in predicting mortality in critically ill patients*. Crit Care Med 43(2):439-444

26. Wolf CA, Wijdicks EFM, Bamlet WR, McClelland RL (2007) Further validation of the FOUR score coma scale by intensive care nurses. Mayo Clin Proc 82(4):435-438

Publisher's note Springer Nature remains neutral with regard to jurisdictional claims in published maps and institutional affiliations. 